\title{
Does perioperative hyperoxia alter the incidence of surgical site infection?
}

Pryor KO, Fahey TJ III, Lien CA, Goldstein PA. Surgical site infection and the routine use of perioperative hyperoxia in a general surgical population. A randomized controlled trial. JAMA 2004;291:79-87.

Background: Surgical site infection (SSI) is a common and serious complication following surgical procedures. A randomized trial published in $2000^{1}$ showed that supplemental oxygen in the perioperative period reduced the risk of SSI, presumably by promoting the bactericidal effect of derived reactive oxygen species in the surgical wound.

Design: In this single-centre randomized controlled trial, patients aged 18 years or more scheduled to undergo laparotomy for a variety of reasons were randomly assigned to receive a fractional inspired concentration of oxygen $\left(\mathrm{FIO}_{2}\right)$ of either 0.80 or 0.35 during the operation and for 2 hours thereafter. Patients were excluded if they had $\mathrm{FIO}_{2}$ requirements greater than 0.35 , severe chronic obstructive pulmonary disease or hemodynamic instability, had previously received bleomycin therapy or were moribund.

The primary outcome measure was SSI within 14 days after surgery as defined by a clinical assessment meeting explicit predefined criteria for SSI and a need for an intervention to manage infection (e.g., antibiotics, opening of the wound). Although changes in the $\mathrm{FIO}_{2}$ were permitted when dictated by a patient's clinical status, study subjects were analyzed according to the group to which they were initially assigned. Both study subjects and investigators evaluating evidence of SSI were blinded to the treatment assignment.

Results: The study was terminated after an interim analysis of the first 160 patients (of a planned 300) unexpectedly showed a significantly higher rate of SSI in the group receiving an $\mathrm{FIO}_{2}$ of 0.80 than in the group receiving an $\mathrm{FIO}_{2}$ of 0.35 (Table 1). In a multivariate logistic regression model, $\mathrm{FIO}_{2}$ remained a statistically significant predictor of SSI, after adjustment for body mass index, duration of surgery, estimated blood loss and volume of crystalloid infused.

Commentary: This randomized controlled trial concluded that high levels of inspired oxygen in the perioperative period increased the rate of SSI. The strengths of the study include its randomization and absence of losses to follow-up.

However, the conclusions are at odds with the results of a previous trial that showed a high $\mathrm{FIO}_{2}$ to be beneficial. ${ }^{1}$ The earlier study differed in that it included only patients having colorectal resection rather than a variety of procedures; subjects also had a higher operative mortality rate and length of hospital stay despite a lower overall incidence of SSI $(8.2 \%$ v. $18.1 \%$ in the present study). Furthermore, in the present study, obesity was not a predictor of SSI, a finding that is also inconsistent with the literature. ${ }^{2}$ Although it is not clear how these differences might account for the opposite conclusions, they do suggest fundamental dissimilarities between the 2 studies in both the populations studied and the management approaches used.

The proposed biologic mechanisms explaining the effect of high $\mathrm{FIO}_{2}$ on SSI cannot account for the large and unexpected adverse effect of high $\mathrm{FIO}_{2}$ observed in this study. It may be that supranormal arterial oxygen tension has beneficial effects on certain subgroups of patients and deleterious effects on others. By raising the possibility of an adverse effect of high $\mathrm{FIO}_{2}$ on some surgical patients, this study argues against the routine implementation of this strategy until its risks and benefits are better understood.

Practice implications: In patients undergoing major abdominal surgery, oxygen should continue to be administered with the goals of maintaining adequate hemoglobin oxygen saturation and ensuring adequate oxygen transport, rather than achieving supranormal levels of arterial oxygen tension.

\section{David R. Urbach}

Departments of Surgery and of Health Policy, Management and Evaluation University of Toronto

Toronto, Ont.

\section{References}

1. Greif R, Akça O, Horn EP, Kurz A, Sessler DI. Supplemental perioperative oxygen to reduce the incidence of surgical-wound infection. $N$ Engl $7 \mathrm{Med}$ 2000;342:161-7.

2. Gottrup F. Prevention of surgicalwound infections. N Engl 7 Med 2000; 342:202-4.

Table 1: Postoperative outcomes of patients undergoing major abdominal surgical procedures, according to the randomly assigned $\mathrm{FIO}_{2}$ in the perioperative period*

\begin{tabular}{lccr}
\hline Outcome & $\begin{array}{c}\text { Group with } \mathrm{FlO}_{2} \text { of } 0.35 \\
n=80\end{array}$ & $\begin{array}{c}\text { Group with } \mathrm{FlO}_{2} \text { of } 0.80 \\
n=80\end{array}$ & $p$ value \\
\hline Infection, no. (\%) & $9(11.3)$ & $20(25.0)$ & 0.02 \\
Length of hospital stay after surgery, mean (SD), d & $6.4(4.7)$ & $8.3(7.5)$ & 0.07 \\
Required reoperation, no. (\%) & 0 & $4(5.0)$ & 0.06 \\
Infection location, no. (\% of those with infection) & $5(56)$ & $13(65)$ \\
$\quad$ Wound only & $1(11)$ & $3(15)$ \\
$\quad$ Deep structures only & $3(33)$ & $4(20)$ \\
Wound and deep structures & & 0.84 \\
\hline
\end{tabular}

Note: $\mathrm{FIO}_{2}=$ fractional inspired concentration of oxygen.

*Adapted from Pryor KO, et al. Surgical site infection and the routine use of perioperative hyperoxia in a general surgical population. A radapted from Pryor KO, et al. Surgical site infection
randomized controlled trial. JAMA 2004;291:79-87. 